\title{
Centrosome RNA: A Molecular Basis for Non-Equivalence of Triplets in Centrioles and Centrosomes?
}

\author{
Marco Regolini* \\ Audiologic, Department of Bioengineering and Mathematical Modeling via De Amicis 25I-20123 Milano, Italy
}

\begin{abstract}
Works have been published about RNA in centrioles, basal bodies and centrosomes but the issue has never been faced and examined in depth. Indeed RNA may really represent the informational molecule that turns the sophisticated architecture of these organelles into highly directional organizing centers: RNA may be the Molecular Basis for NonEquivalence of the centriolar circumferential polarity and the foundation of chirality of centrioles, the most probable organizers of bilateral symmetry in Metazoa.
\end{abstract}

Keywords: Centriole; Centrosome; Basal body

\section{Introduction}

Centrioles are cylindrical organelles, geometrically built by 9 -foldsymmetric triplets [1] each composed of three (named A, B and C) parallel-linked microtubules. Centrioles are equipped with a stock of other scaffolding, highly conserved, proteins, more than 200: the unicellular green alga Chlamydomonas, separated from Mammals by more than $10^{9}$ years of evolution, shares more than $75 \%$ identity with some human centriolar proteins. Basal bodies, at the base of eukaryotic cilia and flagella, are centrioles; the centrosome (the only organelle present in interphase-G1 cells in single copy, like the nucleus) is basically composed of two orthogonal centrioles (besides some dozen proteins). What is the role of such complex (and costly) machines? Centrioles and centrosomes participate in chromosome separation, however they are dispensable: plants and fungi lack centrioles but perform mitosis and meiosis.

Are centrioles necessary only to organize, assemble and manage eukaryotic cilia and flagella? The main functional difference between eukaryotic and prokaryotic flagella (the latter powered by advanced molecular engines capable of realizing clockwise and counter-clockwise rotations) is the to-and-fro rowing movement of the first ones, useful in some respiratory epithelia: too small to explain the evolutionary appearance of these new, completely different and complex structures: flies form hairs and bristles simply through contractile actin bundles; also microvilli and stereocilia are actin based; Daplius, a parasitic protist, builds a flagellum composed of only three microtubules [2] on the contrary, eukaryotic cilia and flagella are stereotypically composed of 9 fold symmetric microtubule doublets and two inner parallel microtubules $(9+2$ structure) requiring a complex motile mechanism and an Intra-Flagellar Transport machinery to carry molecules up and down, copy the nine fold symmetry of basal body triplets and assemble the axonemal architecture.

But then many studies on Protists have highlighted the nonequivalence of centriolar triplets: each triplet appears clearly linked to a distinct component of the cytoskeleton, suggesting an interesting informational role for centrioles and basal bodies: "Several principles of construction of a microscopically small device for locating the directions of signal sources in microscopic dimensions: it appears that the simplest and smallest device that is compatible with the scrambling influence of thermal fluctuations as are demonstrated by Brownian motion is a pair of cylinders oriented at right angles to each other" [3]. "It seems that in Protists and in Metazoa the triplets of basal bodies are not-equivalent" [4]. "Basal bodies and centrioles display structural and functional polarities that play an important role in the spatial arrangement of the cytoskeleton and hence the polarity of the cell"
[5]. "An intrinsically chiral structure, perhaps the centrosome, serves as a template for directing polarity in the absence of spatial cues. Such a template could help to determine left-right asymmetry and planar polarity in development" [6]. "What sub cellular component is responsible for the crucial orientation event that defines "leftward"? One likely possibility is that the coordination of the 3 axes is performed by a cytoskeletal organizing center such as the centriole or basal body" [7]. "To understand cell organization, it will be critical to understand how the different triplets of the centriole come to have distinct molecular identities" [8]. "A ring of nine-singlet microtubules is initiated at metaphase, orthogonal to triplet 8" [9]. The involvement of centrioles in the management (polarization and organization) of the cytoskeleton is evident in many highly organized Ciliates, like Chlamydomonas (two geometrically orthogonal flagella) or Paramecium (about 4,000 cilia organized in 70 longitudinal parallel rows). Centrioles, circumferentially and rotationally polarized by an orderly (genetically coded) sorted sequence of their different triplets, can be orientated in coordinated and shared dispositions to beat in the same direction (this is truly a substantial difference relatively to prokaryotic flagella): Paramecium is capable of producing a coordinated flow of water, containing food particles, directed toward its mouth and sea urchin blastula is free to swim. Then the idea of an informational task played by centrioles seems to hit the target. Prosthecobacter [10] synthetizes the tubulin homologs BtubA and BtubB to build bacterial microtubules that share important features with their eukaryotic counterparts; tubulins are directional molecules of extraordinary importance in evolution: eukaryotic microtubules, robust cylinders made up of 13 parallel filament of dimers of $\alpha$ and $\beta$ tubulin, and widespread from plants to animals, are highly directional structures with a basement (MTOC: MicroTubule Organizing Center) composed of $\gamma$ tubulin and other proteins able to anchor and, above all, drive, tune and adjust the orientation of the arising microtubule. Actin microfilaments are less robust and do not possess easily orientable organizing centers. So microtubules appear as the main directional structures through which eukaryotic cells develop their finely tuned polarity and pluricellular beings acquire the capability of

*Corresponding author: Marco Regolini, Audiologic, Department of Bioengineering and Mathematical Modeling via De Amicis 25I-20123 Milano, Italy, Tel: +3902860474; E-mail: IngRegolini@gmx.com

Received March 24, 2015; Accepted May 20, 2015; Published May 27, 2015

Citation: Regolini M (2015) Centrosome RNA: A Molecular Basis for NonEquivalence of Triplets in Centrioles and Centrosomes?. Mol Biol 4: 125 doi:10.4172/2168-9547.1000125

Copyright: ( 2015 Regolini M This is an open-access article distributed under the terms of the Creative Commons Attribution License, which permits unrestricted use, distribution, and reproduction in any medium, provided the original author and source are credited. 
managing the correct 3D spatial shape (e.g. positioning of constrictionmachinery of actin bundles), the assembly (differential adhesion) of their cells as well as the forecast orientation of the extracellular matrix fibers. By the way, differential adhesivity, oriented elongation and finely tuned polarization play an astonishing fundamental role in shaping amniotes epithelia appendages: mature keratinocytes die, nonetheless, by maintaining their previously precisely localized adhesions, acquired during cytodifferentiation, are able to build extraordinary stereotyped structures like long beaks, colored feathers or sharp claws, made up surprisingly of dead, dehydrated but 3D well-arranged cells. Nonequivalence of triplets appears in Vertebrates too: "Is it possible to confirm this idea that the circumferential, morphological, structural and molecular asymmetry of centrioles can be inferred from Mammals ciliated epithelia. While the circumferential anisotropy of centrioles cannot be ascertained within the centrosome, its existence can be inferred from the properties they express during ciliogenesis, be it the formation of a primary cilium or of bona fide $9+2$ cilia in ciliated epithelia, some of which at least derive directly from the centrioles. As in Ciliates and flagellates, these basal bodies nucleate appendages of various molecular compositions (basal foot, striated rootlets, alarm sheets, etc., which anchor the basal body to the membrane and to the cytoskeleton) and these nucleation's arise at specific sites of the basal body cylinder; in particular, the basal foot is located on triplets 5 and 6 corresponding to the side of the effective stroke of the cilium. What is remarkable is that basal feet develop before the basal bodies reach their membrane site and before they acquire their functional orientation" [4].

In Metazoa two orthogonal (rotationally polarized) centrioles (the centrosome) possess the structure to play the role of a spherical reference system organizer [11] based on two molecular orthogonal protractors/goniometers (one, named mother centriole, responsible for the $\varphi$ coordinate or longitude, the other, named daughter centriole, responsible for the $\theta$ coordinate or latitude) capable of mapping in detail the cortex and membrane of the cell, subdividing them in compartments [12] where both adhesion/constriction factors that assure the correct formation of tissues and receptors (integrins above all) that control and organize the orientation of the fibers in the extracellular matrix (especially collagen) can be differentially positioned: in the proposed geometrical model, the centrosome transmits its spherical polarity to the radial aster of the cytoskeleton, organizing a finely tuned net of directional tracks used by motor proteins (kinesins and dyneins) to precisely deliver their cargoes to the forecast destinations. Indeed plants, fungi and some flatworms (Planaria),lacking centrioles and centrosomes, build only simple laminar and cylindrical tissues, not 3D advanced architectures like semicircular canals, cochleae or eyes, composed of billions of precisely $3 \mathrm{D}$ arranged cells(bilaterally symmetric, as well). The importance of primary cilia in cell signaling confirms this "informational role".

\section{Structural Non-Equivalence of the Triplets}

In their article of 2004 ("The ultrastructure of the Chlamydomonas reinhardtii basal apparatus: identification of an early marker of radial asymmetry inherent in the basal body") Geimer and Melkonian [5] wrote: "Basal bodies and centrioles display structural and functional polarities that play an important role in the spatial arrangement of the cytoskeleton and hence the polarity of the cell... At the distal end of the basal body, exactly at the transition between the microtubular triplets of the basal body and the doublets of the transitional region, a filament of about $10 \mathrm{~nm}$ diameter is attached to the microtubular doublets (rarely triplets) in a rotationally asymmetric pattern linking A-tubules of doublets 7, 8, 9, 1 and 2. From doublet 2, the filament arches back to doublet 7 , passing through the lumen of the basal body. The overall outline of this closed structure resembles that of an (cap less) acorn with its broad base located between doublets 1 and 2, and its pointed tip at doublet 7 . At doublets 2 and 7, the acorn has a prominent knob through which linkage with the A-tubule is established. A second, V-shaped, system of filaments of about $8 \mathrm{~nm}$ diameter is present in this region. Two filaments extend from triplets (rarely doublets) 4 and 5 into the basal body lumen, bisect the lumenal side of the acorn and, in the center of the lumen, connect to another filament that runs perpendicular to the two filaments, extending down from the proximal end of the stellate structure in the transitional region. The filaments extend from triplets 4 and 5 at the level of the acorn and bend downwards with an angle of about $35^{\circ}$ after reaching the lumenal side of the acorn. They continue to the other side, making contact with triplets at the level of the distal connecting fiber attachment. In cross sections, depending on the sectional plane, these filaments can be seen to contact triplets 8, 9 and 1. Most interestingly, both systems of filaments were already present at the distal end of the probasal bodies... Whereas the cartwheel [the ring of SAS-6, the polymer responsible for the 9-fold symmetry, in cross sections resembles a cartwheel] is thought to nucleate the nine fold rotational symmetry of the microtubular triplets, the acorn might play an equally important role imposing rotational asymmetry on the microtubular triplets, perhaps leading to the asymmetric assembly of basal-body-associated fibers and hence cellular asymmetry in general".

What is the biochemical basis of non-equivalence of the triplets and what molecular mechanism can realize such an unusual biological process like the assembly of an ordered, stereotypically sorted, sequence of nine similar but non-equivalent structures? Because each A-microtubule of the triplet assembles in an independent manner, randomly positioned [13] on the ring of SAS-6, how can each triplet acquire its own identity and its correct position within the centriole to generate a forecast ordered rotational polarity? The idea is that the biochemistry of non-equivalence does not consist in the structural difference of the triplets themselves, but in a mechanism able to differentiate and "decorate" nine identical triplets, juxtaposing nine different and orderly sequenced molecular complexes. In other words, non-equivalence is not realized by the tertiary structure of the centriolar blades, rather by their quaternary structure. A ring-shaped polymer is certainly the most likely candidate: a ring containing information (a linearly ordered sequence of equi-spaced markers) and equipped with a handle-stem to be correctly positioned and orientated is the most suitable structure: a ring of RNA?

Dos Santos et al. [14] observe that human centrosomal proteins tend to be larger than generic human proteins: their genes contain more exons (20.3 vs 14.6$)$ and are rich in disordered regions, which cover $57 \%$ of their length, compared to $39 \%$ in the general human proteome; disordered protein domains frequently bind nucleic acids [15]. Nine triplets, if structurally identical, can be easily self-assembled randomly around the SAS- 6 ring that organizes the formation of new centrioles: in a later moment, each of them will acquire its own individuality through the juxtaposition of a ring of an informational macromolecule; so identical triplets become distinguished (and therefore non-equivalent) because of the apposition of nine different markers aligned in an ordered sequence, easily coded, memorized and stored in a linear polymer. In C. reinhardtii the polypeptide VFL1 (Variable number of Flagella), binds only to the triplet $\mathrm{N}^{\circ} 1^{\prime}$ [16]: evidently, its triplet $\mathrm{N}^{\circ} 1$ has its own unique and distinguishable molecular receptor able to recognize and bind VFL1. Similarly, during metaphase, in Chlamydomonas, a ring of nine-singlet microtubules is initiated orthogonally to triplet 8 " [9]. From Protists to Metazoa: Yoshinori and Yukio [17] identified a human centrosomal protein named CLERC (Centrosomal Leucine- 
rich Repeat and Coiled-coil containing protein) which is the ortholog of Chlamydomonas VFL1. They show that bibliography as well as database searches provide evidence that the human proteome contains at least seven centrosomal leucine-rich repeat proteins including CLERC. CLERC and four other centrosomal leucine-rich repeat proteins contain the SDS22-like leucine-rich repeat motifs, whereas the remaining two proteins contain the RI-like and the cysteine-containing leucine-rich repeat motifs. Individual leucine-rich repeat motifs are highly conserved and present in evolutionarily diverse organisms: coiled-coil containing proteins and leucine-/cysteine-rich regions have high propensity to bind with RNA. In conclusion, a ring of RNA is an excellent candidate to realize the non-equivalence of the triplets.

\section{Chirality and Bilateral Symmetry}

My personal opinion is that in Metazoa bilateral symmetry is a fundamental basic property (and an essential requirement) of their locomotive and sensorineural (movement driving) apparatuses: bilateral symmetry is the simplest and the most efficient way to assure balance, drive and control the direction of movements and localize perceived signals (differential stimulation of two equal bilateral effectors or receptors); without bilateral symmetry, the control of balance in fastrunning/-flying animals (prey and predator change directions at a great rate) would be a quite difficult problem; this seems to have been the reason for the extraordinary evolutive success of bilateral symmetry in mobile organisms.

Almost all Metazoa are Bilateria (mirror-symmetric): is it conceivable that in every species, separately each other, genetic developmental programs to build left and right organs have been exactly duplicated with mirror-symmetric, precise geometrical information, or is it more likely that a simpler "left/right translator" has been realized once and for all and then transmitted, highly conserved, to descendant species? After all, bilateral symmetry consists only in the change of the sign $-/+$ of the $\varphi$ coordinate (longitude): this is a simple geometrical concept but very difficult to be realized by evolutionary mechanisms. However, an easy "evolutionary trick" does exist: the overturn of a sequence of markers, orderly assembled on a ring (like a compass dial), automatically "changes" the sign of every angle $\varphi(+/-)$ and a perfect bilateral or mirror symmetry is realized. A simple example: the string "A-H-I-M-O-T-U-V-W-X-Y" on a transparent stripe; by dividing the letters each other with scissor snips $3 / 4$ of the way down from top to bottom, the stripe can be bent to form a planar curved figure (something like a watch dial), and, by joining at the top the letters "A" and "Y", a ring is formed and oriented: the alphabetically ordered string is readable in the clockwise direction; after overturning the ring, on the same plane, the alphabetically ordered string is now readable in the counter-clockwise direction: the overturned ring is mirror (bilaterally) symmetric with respect to the original one. A centriole, polarized with reversed, opposite circumferential sequence (clockwise/ counterclockwise), appears to be the only enantiomorphous cellular organelle, the unique chiral biological structure able to play a geometrical role in left-right patterning: it is a real biological "left/right translator". "Basal bodies/centrioles are an excellent candidate for an intracellular 'F-molecule' $[18,19]$ ].'Centrosomes with opposite polarity of the mother centriole are modelizable as geometrical interfaces which receive the same genetic input (signals with the information for the value of the $\varphi$ coordinate) and translate them symmetrically (output): thus, the same genetic instructions that code for the position of adherens junctions are carried out mirror-symmetrically in cells of right/left side [20]. What mechanism (simple and thus evolutionarily likely) can perform the overturn of a ring to realize opposite polarity? As already seen, O'Toole and Dutcher (2014) [9] identified the specific triplet at which centriole/ basal body duplication occurs in Chlamydomonas: a ring of nine-singlet microtubules is initiated at metaphase, orthogonal to triplet $\mathrm{N}^{\circ} 8$; an active site exists on a mature centriole that drives the building of a new centriole. So, supposing the triplet-polarizing ring has a little handlestem made up by its juxtaposed ends (something like the acceptor stem of a molecule of tRNA) that fits in with the active site that controls the formation of a new centriole, only a small change in its ends is sufficient: if the original stem formed by the join of the ends assumes the form of the capital letter " $F$ ", the chiral molecule hypothesized [18], a symmetric stem like " 7 " can fit in with the active site of its receptor only after an overturn (and an overturn of the whole ring that causes the reversal, clockwise/counter-clockwise, of the circumferential order of markers). Through a small(and thus evolutionarily probable) change in the ends of a linear polymer capable of forming a 3D asymmetric handle-stem, a ring can be overturned. [Obviously, also an opposite process is conceivable: one only, 3D asymmetric, "handle-stem" and two $180^{\circ}$ symmetric receptors (something like one only key and two similar locks rotated of $180^{\circ}$ : to fit in and enter in both, the key must be overturned)]. What mechanism can perform the overturn of a ring to realize opposite polarity? The most likely (known) biologically easy process is a transposition event, followed by independent evolution of the ends: after the transposition, the two loci, having the same sequence and free to evolve separately, maintain the same general order through purifying selection while a few changes in their ends can create two short stems having a symmetric 3D shape. [Also in the opposite case of a $180^{\circ}$ symmetric receptor/active site, a transposition of one exon appears likely: in the coding gene for this receptor, two exons (X and Y) codify for two different parts of the receptor (5'...X-Xxon.... long intron....Y-exon....3"); the transposition of "X" beyond " $Y$ " and a controlled alternative splicing $([\mathrm{X}]=$ non translated $)$ can explain the arising of the $180^{\circ}$ symmetric receptors (right: 5'...X-exon...long intron...Y-exon ...long intron...[X]...3'; left: 5'... [X]...long intron... Y-exon ...long intron...X-exon ....3'); polypeptides coded by $\mathrm{X}$ and $\mathrm{Y}$ exons will appear in different $\left(180^{\circ}\right.$ symmetric) position in the tertiary structure of the receptor. Moreover a mirror symmetric receptor may be made of two different polarized dimers; for example the chain of microtubule filament is a polarized sequence "-... $\alpha \beta \alpha \beta \alpha \beta \ldots+$ ", from which two polarized and $180^{\circ}$ symmetric dimers can be (theoretically) extracted " $-\alpha \beta+$ ", “- $\beta \alpha+$ " : the quaternary structure of receptors is mirror symmetric].

\section{Hypothesis: An RNA Ring Generates the Circumferential Polarity of the Centriole and Its Chirality}

"Structural and genetic studies suggest that asymmetry of the centriole (basal body) plays a critical determining role in organizing the internal organization of algal cells, through the attachment of microtubule rootlets and other large fiber systems to specific sets of microtubule triplets on the centriole...To understand cell organization, it will be critical to understand how the different triplets of the centriole come to have distinct molecular identities" [8].

Geimer and Melkonian [5] highlighted in Chlamydomonas a ringshaped structure resembling that of an acorn with its broad base located between doublets 1 and 2 , and its pointed tip at doublet 7 . At doublets 2 and 7 , the acorn has a prominent knob through which linkage with the A-tubule is established. Dippel identified an RNase sensitive ring in Chlamydomonas basal bodies [21].

Triplets have identical and equivalent primary, secondary and tertiary structures: their non-equivalence likely consists in the collocation and juxtaposition of nine different polyribonucleotidic-marks that can, in turn, bind different proteins (non-equivalence of the quaternary structure). 
A single-stranded filament of RNA, because of its chemicalphysical properties and the frequent modification of the bases, exhibits a variety of thermodynamically favorable secondary double-stranded structures (hairpins, stems, loops, knots, pseudo knots): these, in turn, create distinct 3D tertiary configurations (transfer-RNAs are wellknown examples). A single-stranded RNA ring (memorized linearly in DNA, which encodes nine different molecular labels, in an orderly sequence and equally spaced) possesses all the characteristics to be the molecular platform of the non-equivalence of the nine centriolar blades. A ring of RNA can, in turn, bind different polypeptides to form ribonucleoproteins [22] or various oligosaccharides [23].

\section{Centrosomal RNA}

Already in 1974 Laane and Haugli studied the association of RNA with centrosomes in Physarum, while Hartman et al. confirmed the same finding in Tetrahymena; Dippel [21] identified an RNase sensitive ring in Chlamydomonas basal bodies. Hiedemann et al., reported RNase sensitivity of Chlamydomonas basal bodies; Snyder [24] showed that RNase inhibited microtubule nucleation in PtK1 cells; Alliegro et al., [22,25-27] isolated centrosomal RNAs (cnRNAs), absent from the remaining cytoplasm, in Spisula Solidissima; Chichinadze [28] and colleagues continued the studies on Spisula cnRNAs, focusing attention on a reverse transcriptase domain and a ribonucleoprotein consensus RNA-binding site contained in one of cnRNAs. The importance of RNA in centrosomes has been reaffirmed also by Ishigaki et al. showing that RBM8A/MAGOH heterodimer (a member of exon junction complex on exporting mRNA, required for mRNA splicing, mRNA export and nonsense-mediated mRNA decay) formed a complex in centrosomes.

\section{cnRNA11}

Alliegro and colleagues described many centrosomal RNAs in Spisula solidissima (the Atlantic surf clam, a large bivalve, bilaterally symmetric, mollusk): among them there are two particularly interesting cnRNAs named "cnRNA11" which this analysis deals with; one is long (3863 nucleotides, GenBank: DQ359732) and considered by the author to be an mRNA: "a substantial ORF [Open Reading Frame] was readily mapped in the antisense strand. The predicted polypeptide encoded in this ORF includes a highly conserved 200 amino acidlong reverse transcriptase domain.... In addition, a ribonucleoprotein consensus RNA-binding site (RNP-1) was found downstream from the reverse transcriptase domain." [25]; the other cnRNA11 is short (638 nt, GenBank: DQ363378). Aligning these two sequences (rather the sequences of their coding DNA loci), the long one (from the 2255 to the 2880 nt of its Plus DNA filament) matches and corresponds very well with the sequence from the 630 to the 3 nt of the short one (Minus DNA filament), with clearly reverse alignment (Plus/Minus): however the correspondence is not perfect (93\%), because of 6 insertion/deletions and the change of some nucleotides (8in the 5 ' end of the short RNA11, 2 in its 3' end): these are then two non-identical sequences, coded by two different loci, one, long, in the Plus DNA strand and the other, short, in the Minus DNA strand. The two cnRNA11 loci have evolved independently maintaining their substantial sequence identity, with a few changes allowed by purifying selection. These findings support the hypothesis (at least in Spisula) of a retro-transposition event, which maintains $5^{\prime}>3^{\prime}$ order and requires the intervention of nucleases (RNase-reverse transcriptase) responsible for the alteration of the ends. The ends of the transposed short cnRNA11, different from the ends of the long cnRNA11,could then assemble stems showing 3D bilaterally symmetric shapes: if so, after entering into the active site of the mother centriole, the transposed short cnRNA11 ring is overturned and the sequence of its nucleotides is opposite and reversed in respect to the original long cnRNA11:these observations agree with the previous considerations on small(thus evolutionarily probable) changes in the ends necessary for an RNA ring to create two short 3D mirrorsymmetric stems.

It is convenient to fix two points of reference in the long cnRNA11 (Figure 1): "START" is the beginning of the region of similarity between the two cnRNA11 that, on the Plus DNA strand coding for the long cnRNA11, has the following sequence (5'>3'): “TGACG" (2255 to 2259 nt); "FINISH" is the end of the region of similarity, that, on the Plus DNA strand coding for the long cnRNA11, has the sequence ( $5^{\prime}>3$ '): "GCTAG" (2876 to $2880 \mathrm{nt}$ ). The same sequences are readable on the Minus DNA (coding) strand of the short cnRNA11, in the same direction 5'>3'.

Let's now examine the nucleotides which follow the "FINISH" sequence in the long cnRNA11 Plus (coding) DNA strand: the sequence 5'>3' "TTGAGTTA" is similar to the 5'>3' sequence that precedes the "START" in the short cnRNA11 Minus (coding) DNA strand ("GATGGAAG"): reading this last sequence in direction 3'>5' a consensus sequence "A G G/T T A" appears. Note that, aligning the ends of a string bent into a ring (something like the capital letter " $U$ "), one end is upturned relative to the other: to read both the " $U$ "-stems from the top to the bottom, they must be read in the opposite direction: the $5^{\prime}$ end is read in direction $5^{\prime}>3^{\prime}$ whereas the $3^{\prime}$ end must be read in direction 3'>5'. So, a consensus sequence (5'>3') “AGTTA" is at the 3 ' end (immediately after the FINISH) of the long cnRNA11 Plus (coding) DNA filament, while a similar sequence "AGGTA" (3'>5') is at the 5' end (immediately before the START) of the short cnRNA11 Minus (coding) DNA filament.

This consensus sequence (very similar to that of the short variable loop of tRNA) can generate a similar bulge with the concavity facing the opposite stem-end. When the transcribed consensus sequence "UC C/A A U" (and its knob) is on the same side of the handle-stem (showing similar orientation) the two cnRNA11 rings are evidently overturned and bilaterally symmetric (Figure 2). When the stems of both short and long cnRNA11 show the consensus sequence on the same side, the two rings are overturned: "START" and "FINISH" sequences are mirror symmetric.

As already said, it is also conceivable another likely scenario in which right and left mother centrioles possess different, $180^{\circ}$ symmetric, $3 \mathrm{D}$ receptors/active sites to assembly and patterning their daughters, forcing the RNA ring to overturn: the right mother centriole receptor can interact exclusively with the "right" centrosomal RNA stem (i.e. the 3D shape of the stem when the "head" face of the ring is turned up) to build only a new right centriole, whereas left mother centrioles interact exclusively with "left" centrosomal RNA stems (i.e. the 3D shape of the stems when the "tail" face of the ring is turned up);as already said, O'Toole and Dutcher [9] identified the specific triplet at which centriole/ basal body duplication occurs in Chlamydomonas, showing that a ring of nine-singlet microtubules is initiated at metaphase, orthogonal to triplet $\mathrm{N}^{\circ} 8$ : if this mother centriole "active site" exists in two $180^{\circ}$ symmetrical 3D opposite configurations capable of overturning the RNA ring, only one cnRNA is necessary, equipped with a $3 \mathrm{D} 180^{\circ}$ symmetric stem.

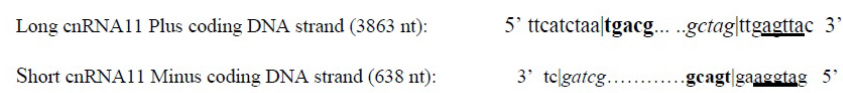

Figure 1: bold: START sequence; italics: FINISH sequence; underlined: consensus sequence 


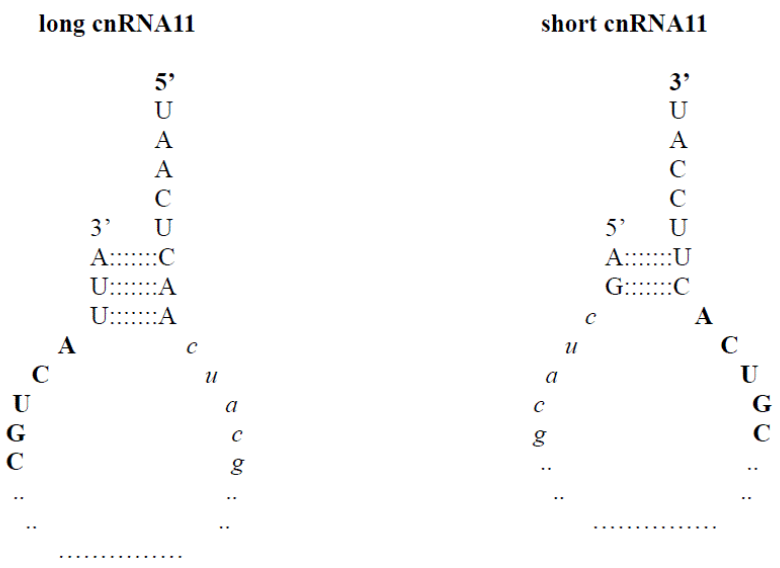

Figure 2: cnRNA11 ring-stems

\section{Conclusions}

The hypothesis that a transposition event has originated in $S$. Solidissima the short cnRNA11 appears likely; this CNrna11-ring, in turn, when used in an overturned fashion, generates a mirror-symmetric decoration of the mother centriole responsible for the $\varphi$ coordinate of a spherical reference system: this might effectively represent a simple and evolutionarily likely, biochemical basis of metazoan bilateral symmetry. In different clades different ways may be followed to reach the same result: one cnRNA only and two symmetrical different right/ left receptors on the mother centrioles carry out the overturn of the cnRNA ring. The above analysis shows that well-known molecular and biological mechanisms are capable of easily realizing the circumferential non-equivalence of the triplets and the chirality of the mother centriole.

\section{Reference}

1. Guichard P, Hachet V, Majubu N, Neves A, Demurtas D, et al. (2013) Native architecture of the centriole proximal region reveals features underlying its 9-fold radial symmetry. Curr Biol 23: 1620-1628.

2. Lodish AB (2012) Molecular Cell Biology. New York, W.H. Freeman \& Co. (section 19.4, Cilia and Flagella)

3. Albrecht-Buehler G (1981) Does the geometric design of centrioles imply their function? Cell Motil 1: 237-245.

4. Beisson J, Jerka-Dziadosz M (1999) Polarities of the centriolar structure: morphogenetic consequences. Biol Cell 91: 367-378

5. Geimer S, Melkonian M (2004) The ultrastructure of the Chlamydomonas reinhardtii basal apparatus: identification of an early marker of radial asymmetry inherent in the basal body. J Cell Sci 117: 2663-2674.

6. Xu J, Van Keymeulen A, Wakida NM, Carlton P, Berns MW, et al. (2007) Polarity reveals intrinsic cell chirality. Proc Natl Acad Sci U S A 104: 92969300.

7. Vandenberg LN, Levin M (2009) Perspectives and open problems in the early phases of left-right patterning. Semin Cell Dev Biol 20: 456-463.

8. Marshall WF (2012) Centriole asymmetry determines algal cell geometry. Curr Opin Plant Biol 15: 632-637.

9. O'Toole ET, Dutcher SK (2014) Site-specific basal body duplication in Chlamydomonas. Cytoskeleton (Hoboken) 71: 108-118.

10. Pilhofer M, Ladinsky MS, McDowall AW, Petroni G, Jensen GJ (2011)
Microtubules in bacteria: Ancient tubulins build a five-protofilament homolog of the eukaryotic cytoskeleton. PLoS Biol 9: e1001213.

11. Regolini M (2014) The Spherical Reference System of Metazoan Cells. J Phys Chem Biophys 4:151.

12. Dodgson J, Chessel A, Yamamoto M, Vaggi F, Cox S, et al. (2013) Spatial segregation of polarity factors into distinct cortical clusters is required for cell polarity control. Nat Commun 4: 1834.

13. Guichard P, Chrétien D, Marco S, Tassin AM (2010) Procentriole assembly revealed by cryo-electron tomography. EMBO J 29: 1565-1572.

14. Dos Santos HG, Abia D, Janowski R, Mortuza G, Bertero MG, et al. (2013) Structure and non-structure of centrosomal proteins. PLoS One 8: e62633.

15. Vuzman D, Levy $Y(2012)$ Intrinsically disordered regions as affinity tuners in protein-DNA interactions. Mol Biosyst 8: 47-57.

16. Silflow CD, LaVoie M, Tam LW, Tousey S, Sanders M, et al. (2001) The Vfl1 Protein in Chlamydomonas localizes in a rotationally asymmetric pattern at the distal ends of the basal bodies. J Cell Biol 153: 63-74.

17. Yoshinori M, Yukio O (2010) CLERC and centrosomal leucine-rich repeat proteins. Cent Eur J Biol 5: 1-10.

18. Brown NA, Wolpert $L$ (1990) The development of handedness in left/right asymmetry. Development 109: 1-9.

19. Levin M, Palmer AR (2007) Left-right patterning from the inside out: widespread evidence for intracellular control. Bioessays 29: 271-287.

20. Regolini MF (2013) Centrosome: is it a geometric, noise resistant, 3D interface that translates morphogenetic signals into precise locations in the cell? Ital J Anat Embryol 118: 19-66.

21. Dippell RV (1976) Effects of nuclease and protease digestion on the ultrastructure of Paramecium basal bodies. J Cell Biol 69: 622-637.

22. Alliegro MC (2001) The centrosome and spindle as a ribonucleoprotein complex. Chromosome Res 19: 367-376.

23. Liu L, Botos I, Wang Y, Leonard JN, Shiloach J, et al. (2008) Structural basis of toll-like receptor 3 signaling with double-stranded RNA. Science 320: 379-381.

24. Snyder JA (1980) Evidence for a ribonucleoprotein complex as a template for microtubule initiation in vivo. Cell Biol Int Rep 4: 859-868.

25. Alliegro MC, Alliegro MA, Palazzo RE (2006) Centrosome-associated RNA in surf clam oocytes. Proc Natl Acad Sci USA 103: 9034-9038.

26. Alliegro MC, Alliegro MA (2008) Centrosomal RNA correlates with intron-poor nuclear genes in Spisula oocytes. Proc Natl Acad Sci U S A 105: 6993-6997.

27. Alliegro MA, Henry JJ, Alliegro MC (2010) Rediscovery of the nucleolinus a dynamic RNA-rich organelle associated with the nucleolus, spindle, and centrosomes. Proc Natl Acad Sci U S A 107: 13718-13723.

28. Chichinadze K (2013) RNA in centrosomes: structure and possible functions Protoplasma 250:397-405. 